\title{
EOLÉTIOA volume 38, 2013 química \\ CONCENTRAÇÃO DE MATERIAL PARTICULADO E COMPOSIÇÃO ELEMENTAR NO AEROSSOL ATMOSFÉRICO DE UMA REGIÃO DE PRODUÇÃO DO AÇO NO ESTADO DE MINAS GERAIS-BRASIL
} Artigo/Article

\author{
Viviane Macedo Reis Araújo ${ }^{1}$, Gabriela von Rückert ${ }^{1}$, Maria Ângela de B. C. Menezes ${ }^{2}$, Humberto Lopes \\ Santos $^{3}$, Adriana Rocha de Souza Drumond ${ }^{1}$
}

\begin{abstract}
${ }^{1}$ Programa de Mestrado em Engenharia Industrial do Centro Universitário do Leste de Minas Gerais (UNILESTE). Coronel Fabriciano, MG, Brasil viviane.macedoreis@gmail.com gruckert@homail.com.br adrianarochadrumond@ hotmail.com.²Centro de Desenvolvimento da Tecnologia Nuclear/Comissão Nacional de Energia Nuclear (CDTN)/CNEN) Laboratório de Ativação Neutrônica,Belo Horizonte, MG, Brasil menezes@cdtn.br. ${ }^{3}$ Coordenação de Meio Ambiente e Qualidade - Celulose NipoBrasileira (CENIBRA S.A) Belo Oriente, MG, Brazil humberto.santos@cenibra.com.br
\end{abstract}

Os municípios de Ipatinga (IPA) e Coronel Fabriciano (FAB) estão inseridos na Região Metropolitana do Vale do Aço (RMVA). Nesta região estão localizadas duas indústrias siderúrgicas e uma indústria de extração de celulose. Neste estudo, foram obtidas as concentrações de Partículas Totais em Suspensão (PTS), Partículas Inaláveis com tamanho aerodinâmico de até $10 \mu \mathrm{m}\left(\mathrm{MP}_{10}\right)$ por gravimetria, utilizando amostradores manuais HI-VOL (AGV), além da concentração de elementos químicos no material particulado em IPA e FAB de maio a novembro de 2010. A concentração de elementos no material particulado amostrado foi obtida por meio das técnicas de Análise por Espectrometria de Absorção Atômica: $\mathrm{Al}, \mathrm{Ca}, \mathrm{Cu}, \mathrm{Cd}, \mathrm{Mg}, \mathrm{Mn}, \mathrm{Ni}, \mathrm{Pb}, \mathrm{Sn}, \mathrm{Ti}, \mathrm{Zn}$, Ativação Neutrônica Instrumental (método k0): As, Br, Ce, Co, $\mathrm{Cr}$, Cs, Fe, Hf, La, Sb, Sc, Sr, Th, K, Zn, Zr, e o Na por Espectrofotometria de Emissão de Chama. A qualidade do ar foi avaliada com base nas concentrações de PTS e $\mathrm{MP}_{10}$ de acordo com a Resolução CONAMA 03/1990 e elementos pelos padrões da Organização Mundial da Saúde (OMS). Observou-se que em FAB a concentração de PTS e principalmente $\mathrm{MP}_{10}$ se apresentou com maior variação e alcançou valores superiores ao limite estabelecido pela Resolução CONAMA mais frequentemente se comparado ao ponto em IPA. A qualidade do ar se apresentou em geral BOA para PTS e $\mathrm{MP}_{10}$ em ambos os locais amostrados. Os elementos $\mathrm{Sn}, \mathrm{Ca}, \mathrm{Fe}, \mathrm{Pb}, \mathrm{Na}, \mathrm{K}, \mathrm{Zn}$ e $\mathrm{Al}$ nas PTS e $\mathrm{MP}_{10}$ em ambos os municípios foram os mais abundantes. Fe e Sn se apresentaram acima do limite estabelecido pela norma OMS, assim como $\mathrm{Cr}, \mathrm{Pb}$ e As. As concentrações mais elevadas de PTS, $\mathrm{MP}_{10}$ e elementos foram registradas nos meses secos e frios. De acordo com os resultados apresentados, verificou-se uma forte correlação entre a composição e concentrações de elementos e as atividades siderúrgicas e de tráfego de veículos na região.

Palavras-chave: elementos tóxicos, material particulado, qualidade do ar, vale do aço.

\section{ABSTRACT}

Ipatinga (IPA) and Coronel Fabriciano (FAB) cities are located in Vale do Aço, a metropolitan area and the largest industrial area in Minas Gerais state, where there are two steel mills and one pulp mill, as well as other micro and small factories. Due to industrialization, population growth, technological development and intense vehicle traffic in the region, the air pollution has been increasing. Air pollution can result in public health problems, thus it is necessary to quantify the particulate matter concentration and the toxic metals and trace elements associated in this region. This study evaluated the concentration of the total suspended particles (TSP), inhalable particles with aerodynamic size up to $10 \mu \mathrm{m}\left(\mathrm{PM}_{10}\right)$ by HI-VOL manual samplers 
in IPA and FAB, from May to November 2010. Metals and trace elements were determined in the $\mathrm{PM}_{10}$ and TSP samples. Neutron Activation Analysis, k0-method,was applied and determined the elements As, Br, $\mathrm{Ce}$, $\mathrm{Co}, \mathrm{Cr}, \mathrm{Cs}, \mathrm{Fe}, \mathrm{Hf}, \mathrm{La}, \mathrm{Sb}, \mathrm{Sc}, \mathrm{Sr}, \mathrm{Th}, \mathrm{K}, \mathrm{Zn}, \mathrm{Zr}$; Atomic Absorption Spectrophotometry, Al, Ca, Cu, Cd, $\mathrm{Mg}, \mathrm{Mn}, \mathrm{Ni}, \mathrm{Pb}, \mathrm{Sn}, \mathrm{Ti}, \mathrm{Zn}$, and Flame Emission Photometry, Na. The air quality was assessed based on the concentrations of TSP and PM10 according to CONAMA Resolution 03/1990 and elements by the standards of the World Health Organization (WHO). It was observed that in the FAB concentration of TSP and PM10 mainly performed with greater variation and reached the upper limit established by CONAMA figures most often compared to the point in IPA. Air quality is generally presented GOOD for TSP and MP10 in both sampling sites. The Sn, Ca, Fe, Pb, elements Na, K, Zn and Al in TSP and PM10 in both counties were the most abundant. Fe and $\mathrm{Sn}$ were above the limit set by the WHO standard, as well as $\mathrm{Cr}, \mathrm{Pb}$ and As The highest concentrations of TSP, PM10 and elements were observed during the dry and cold months. The high concentration results of $\mathrm{Fe}$ and $\mathrm{Sn}$ suggested that the sources of emissions were hematite transport and steel mill. It was found a strong correlation between elements concentrations and the steel factory activities and the vehicle traffic in this region.

Key-words: toxic elements, particulate matter, air quality, steel mill.

INTRODUÇÃO

A RMVA é um pólo industrial de Minas Gerais, onde estão localizadas duas indústrias siderúrgicas (Usiminas e APERAM) e uma indústria de extração de celulose (Cenibra), além de micro e pequenas empresas.

Os municípios Ipatinga (IPA) e Cel. Fabriciano (FAB), juntamente com Timóteo e Santana do Paraíso, constituem a RMVA [1]. Tratase de uma aglomeração urbana com aproximadamente 468.378 habitantes. Somadas as populações dos outros 11 municípios, que formam o colar metropolitano, a região atinge mais de 600 mil habitantes [2].

A economia de IPA baseia-se principalmente na siderurgia. IPA passa por um processo de diversificação econômica, com a implantação de um distrito industrial que vem estimulando o empreendedorismo e a abertura de novas plantas industriais.

Já a economia de FAB está ligada ao comércio. Por se localizar entre os municípios de Timóteo e IPA, onde existem as principais indústrias siderúrgicas de Minas Gerais (MG). Mesmo não possuindo instaladas indústrias de grande porte em sua área de abragência, FAB pode apresentar uma elevada poluição atmosférica, que pode ser representada pela carga de material particulado (MP) advindo das indústrias siderúrgicas das outras cidades e do crescente tráfego de veículos.

As principais fontes de poluição atmosférica na RMVA são siderurgia, indústrias de transformação, queimadas e tráfego de veículos, o que torna importante a quantificação de elementos químicos que se aglomeram no MP. Os mesmos podem ser classificados em partículas totais em suspensão (PTS) e partículas inaláveis $\left(\mathrm{MP}_{10}\right)(\leq 10$ $\mu \mathrm{m})$. Os $\mathrm{MP}_{10}$, quando inalados, podem se alojar nos alvéolos pulmonares, causando doenças respiratórias, cardiovasculares, alergias e câncer. Com isso, o número de internações nos hospitais da região, pode estar relacionado à quantidade de $\mathrm{MP}$ oriundo de atividades industriais e de deslocamento.

No Brasil o padrão legal para poluição atmosférica é baseado na Resolução CONAMA $\mathrm{n}^{\circ}$ 03 de 1990, ou seja, valores máximos de $150 \mu \mathrm{g} . \mathrm{m}^{-3}$ para MP em 24 horas de amostragem, assim como o Índice de Qualidade do Ar (IQar) (Quadro 01). Entretanto, a Organização Mundial da Saúde (OMS) estabelece limites máximos para ambos de $50 \mu \mathrm{g} . \mathrm{m}$ 3. Associados a essas partículas podem ser encontrados elementos tóxicos na forma de óxidos.

O objetivo deste estudo foi avaliar e classificar a qualidade do ar em termos das concentrações de MP: PTS, MP $_{10}$ e elementos agregados aos mesmos, nos dois municípios supracitados do estado de MG.

\section{MATERIAIS E MÉTODOS}

O monitoramento foi realizado por meio da instalação temporária de dois kits de amostradores ativos de grande volume (AGV - HI-VOL) por gravimetria $[3,4,5]$.

Um dos kits foi instalado dentro da área do Centro Universitário do Leste de Minas Gerais (UNILESTE) em FAB (campus Caladinho) e outro em IPA (campus Bom Retiro), dentro da esfera de influência das possíveis fontes de emissão. 
As técnicas utilizadas na determinação da concentração de elementos químicos no MP foi espectrometria de absorção atômica para $\mathrm{Al}, \mathrm{Ca}, \mathrm{Cu}$, $\mathrm{Cd}, \mathrm{Mg}, \mathrm{Mn}, \mathrm{Ni}, \mathrm{Pb}, \mathrm{Sn}, \mathrm{Ti}, \mathrm{Zn}$, ativação neutrônica instrumental (método k0) para $\mathrm{As}, \mathrm{Br}, \mathrm{Ce}, \mathrm{Co}, \mathrm{Cr}$, $\mathrm{Cs}, \mathrm{Fe}, \mathrm{Hf}, \mathrm{La}, \mathrm{Sb}, \mathrm{Sc}, \mathrm{Sr}, \mathrm{Th}, \mathrm{K}, \mathrm{Zn}, \mathrm{Zr}$ e espectrofotometria de emissão de chama para o $\mathrm{Na}$ $[6,7,8,9,10,11,12]$.

As coletas consistiram em três amostras semanais de cada parâmetro, no ano de 2010 nos meses de maio, junho, julho e agosto. Nos meses de setembro, outubro e novembro, as amostras foram coletadas uma vez em cada semana, ambas com 24 horas de exposição. As coletas foram realizadas nos dois pontos simultaneamente.

Os tratamentos estatísticos foram média aritmética e desvio padrão, a partir dos valores diários das concentrações. As diferenças estatísticas entre os meses de estação seca e de transição e entre os pontos de amostragem foram avaliadas utilizando TESTE-T ou Wilcoxon com grau de significância a $5 \%[10]$.

A comparação das concentrações dos elementos em ambos os municípios foi realizada por Wilcoxon. A relação entre as concentrações de PTS e $\mathrm{MP}_{10}$ foram avaliadas por correlação de Pearson [8].

Para a classificação da qualidade do ar e cálculo do IQAR foram utilizados os dados brutos diários [13].

\section{RESULTADOS E DISCUSSÕES}

\section{Concentração de PTS e MP 10}

Observou-se que em FAB a concentração de PTS e $\mathrm{MP}_{10}$ se apresentou com maior variação e alcançou valores superiores ao limite estabelecido pela Res. CONAMA mais frequentemente do que no ponto em IPA. Foram observadas altas concentrações de PTS em FAB no dia 09 de junho $\left(165 \mu \mathrm{g} . \mathrm{m}^{-3}\right)$ e em IPA no dia 26 de julho $\left(141 \mu \mathrm{g} . \mathrm{m}^{-}\right.$ $\left.{ }^{3}\right)$ e para $\mathrm{MP}_{10}$ em FAB $\left(167 \mu \mathrm{g} \cdot \mathrm{m}^{-3}\right)$ neste mesmo dia.

Esta concentração mais elevada nestes dias, pode ter ocorrido por influência de incêndio florestal numa área de preservação próxima aos pontos amostrados. Incêndios florestais se enquadram em fontes naturais de material particulado [14].

As concentrações mais elevadas de PTS, $\mathrm{MP}_{10}$ e elementos foram registradas nos meses secos e frios (junho a agosto), devido aos baixos índices de precipitação úmida e ocorrência de calmaria $[15,16$, 17].

Em FAB as médias das concentrações de PTS se apresentaram com maior variação e média mais elevada nos meses entre maio e agosto (estação seca) se comparado com o período de transição (setembro a novembro) (Teste-T, $\mathrm{N}=21 ; \mathrm{p}=0,0108$ ). Já em IPA, apesar da ligeira variação, as médias das concentrações não mostraram diferenças significativas entre os períodos de seca e transição (Teste- $\mathrm{T}, \mathrm{N}=18 ; \mathrm{p}=0,0714$ ), ou seja, neste ponto os valores das concentrações foram mais homogêneos do que FAB durante os dois períodos (TAB. 01).

Apesar de não apresentar diferença significativa entre os valores médios de PTS entre FAB e IPA (Teste-T , N=39; $\mathrm{p}=0,4852$ ), FAB foi o único ponto onde o padrão secundário do CONAMA foi excedido. Esta concentração mais elevada pode ter sido devida à influência do tráfego de veículos próximo ao local de amostragem em FAB. A menos de $200 \mathrm{~m}$ deste ponto há uma rodovia importante para o transporte da região, onde pode haver significativa ressuspensão de poeira de caminhões pesados, escapamentos de gases nos canos dos veículos, os quais são caracterizados como uma forma de fonte móvel de emissão atmosférica [10, $18]$.

As concentrações de $\mathrm{MP}_{10}$ em $\mathrm{FAB}$ variaram entre 12 a $167 \mu \mathrm{g} \cdot \mathrm{m}^{-3}$, apresentando média de concentração maior do que IPA, 43,95 e 25,11 $\mu \mathrm{g}$.m 3 , respectivamente (Teste de Wilcoxon, $\mathrm{N}=39$; $\mathrm{p}=0,0171$ ) durante todo o período (TAB. 02).

Também em FAB a concentração $\mathrm{MP}_{10}$ apresentou valores maiores no período de estação seca se comparado com o período de transição (Teste- $\mathrm{T}, \mathrm{N}=21 ; \mathrm{p}=0,0002$ ). Isto pode ser explicado pelas variações nas condições metereológicas [19].

Em IPA os padrões $\left(150 \mu \mathrm{g} \cdot \mathrm{m}^{-3}\right)$ da Res. CONAMA, não foram excedidos durante o período de amostragem. Além disso, os valores de concentração foram relativamente baixos e não apresentaram diferenças estatisticamente significativas entre as estações de seca e transição (Teste-T, $\mathrm{N}=18 ; \mathrm{p}=0,1891$ ). Isto indica uma maior estabilidade nas concentrações de $\mathrm{MP}_{10}$ em IPA (TAB. 02). Esta maior estabilidade pode ser devido à menor quantidade de fontes de emissão neste ponto. Sobretudo, o tráfego de veículos próximo a este ponto de amostragem foi relativamente menor do que FAB. 


\section{Concentração de elementos nas PTS e MP $\mathbf{P}_{10}$}

As concentrações significativas foram para os elementos Fe $\left(77,3 \mu \mathrm{g} . \mathrm{m}^{-3}\right), \mathrm{Ca}\left(25,7 \mu \mathrm{g} . \mathrm{m}^{-3}\right), \mathrm{Na}$ $\left(24,0 \mu \mathrm{g} \cdot \mathrm{m}^{-3}\right)$ e Sn $\left(12,1 \mu \mathrm{g} \cdot \mathrm{m}^{-3}\right)$ em FAB. Em IPA, estas foram para $\mathrm{Fe}\left(35,7 \mu \mathrm{g} . \mathrm{m}^{-3}\right)$ e $\mathrm{Sn}\left(17,2 \mu \mathrm{g} \cdot \mathrm{m}^{-3}\right)$. Isto podem ser devido às emissões no transporte de hematita, decorrente da proximidade com as usinas, ressuspensão do solo e tráfego de veículos [10].

Foram observadas as correlações significativas entre as PTS e os elementos em FAB (TAB. 03). Entre as boas correlações evidenciadas neste estudo ( $\mathrm{r}>0,70)$, foram observadas correlações altamente significativas entre PTS e os elementos $\mathrm{Cu}, \mathrm{Mn}, \mathrm{Zn}, \mathrm{Ca}, \mathrm{Mg}$, Sn e Al. Em IPA, foram observadas correlações de PTS entre os elementos $\mathrm{Cu}, \mathrm{Fe}, \mathrm{Mn}, \mathrm{Zn}, \mathrm{Mg}, \mathrm{Cr}, \mathrm{Na}, \mathrm{Sc}, \mathrm{Th}, \mathrm{As}$ e $\mathrm{Br}$ (TAB. 04). Isto evidencia que mesmo apresentando variações de concentração ou fontes distintas, as PTS estão fortemente associadas a estes elementos.

A correlação elevada entre PTS/Fe $(r=0,79)$ em IPA pode ser explicado pela proximidade de sua fonte de emissão, se apresentando como poluente primário associado às partículas maiores [12].

A influência de fontes móveis para emissão de PTS é predominante em FAB, pois também foram encontradas correlações positivas entre PTS/Ca $(r=0,89), \quad$ Al $(r=0,84)$ e Sn $(r=0,83)$. Entretanto, em IPA as boas correlações das PTS com $\mathrm{Sc}, \mathrm{Fe}, \mathrm{Na}, \mathrm{Br}, \mathrm{Cr}$, As e Th, fortalece hipótese de este ponto sofrer mais influência das fontes fixas do que de fontes móveis, pois os elementos traços Sc, $\mathrm{Cr}$, As e Th podem ser advindos exclusivamente da indústria siderúrgica próxima ao ponto de amostragem.

A quantidade de correlações dos elementos nas PTS se torna um problema, pois estudos indicaram que $70 \%$ nas PTS são $\mathrm{MP}_{10}$ [12].

Nas $\mathrm{MP}_{10}$ de IPA foram observadas menores quantidades de correlações significativas entre os elementos analisados em relação a FAB. Esse ponto apresenta uma menor quantidade de fontes de emissões e as concentrações de $\mathrm{MP}_{10}$ nos filtros também foram menores (Wilcoxon, $\mathrm{N}=39$; $\mathrm{p}=0,0171)$. Isto pode ser devido à predominância de fontes distintas de emissão de partículas em FAB. Em IPA, os elementos mais fortemente correlacionados com as partículas são principalmente provenientes de fontes fixas (siderúrgica), enquanto que em $\mathrm{FAB}$, os elementos que se correlacionam melhor são provenientes de fontes móveis e estacionárias.
Ao se confrontar as concentrações de elementos com as concentrações de $\mathrm{MP}_{10}$, foram encontradas fortes correlações em FAB $(r>0,90)$ (TAB. 05) e IPA $(r>0,80)$ (TAB. 06), entre $\mathrm{MP}_{10} \mathrm{e}$ $\mathrm{Mn}, \mathrm{Ca}, \mathrm{Mg}, \mathrm{K}$. Foi possível observar também boas correlações $(r>0,75)$ entre $\mathrm{MP}_{10}$ e $\mathrm{Cu}, \mathrm{Fe}, \mathrm{Zn}, \mathrm{Cr}$, $\mathrm{Sn}, \mathrm{Na}, \mathrm{La}, \mathrm{Sb}$, As e Br em FAB. A presença de tais elementos se apresentaram associada com as partículas que compõem o $\mathrm{MP}_{10}$ na área de estudo, pode ser explicado pelo fato dos mesmos apresentarem alta capacidade de se agregar as partículas menores que $10 \mu \mathrm{m}$, assim eles podem ser inalados em diferentes formas químicas, dependendo de suas características, principalmente na forma de óxidos [11].

As fortes correlações entre $\mathrm{MP}_{10}$ e $\mathrm{Fe}$ em FAB $(r=0,92)$ (TAB. 05) e com $\mathrm{Sn}(\mathrm{r}>0,80)$ este também com PTS apenas em FAB, pode ser em decorrência do transporte de minério de ferro pela rodovia e ferrovia que cortam o município, a correlação significativa entre $\mathrm{MP}_{10} / \mathrm{Fe}$ para este ponto pode ser explicado pela presença de $\mathrm{Fe}$ em diversas fontes e em partículas menores, ou seja, estas com presença de óxido de ferro agregadas podem ter sido transportadas por longas distâncias em decorrência das interpéries [18].

Embora alguns deles sejam essenciais ao corpo humano ( $\mathrm{Zn}$ e $\mathrm{Fe}$ ) se inalados em altas concentrações, ou mesmo na forma de vapor, podem ser prejudiciais à saúde da população [11].

Sendo assim é importante destacar a boa correlação entre $\mathrm{PM}_{10} / \mathrm{Cr}(\mathrm{r}=0,84)$ em $\mathrm{FAB}$ (TAB. 05), pois este elemento é altamente tóxico, principalmentes nos estados de oxidação +3 e +6 . O $\mathrm{Cr}^{+3}$ tem ocorrência natural, enquanto que o $\mathrm{Cr}^{+6}$, $\mathrm{Cr}^{+2}$ e $\mathrm{Cr}^{0}$ advém de processos industriais. A forma bivalente é facilmente oxidada para a forma trivalente pelas reações atmosféricas [20].

Além disso, o $\mathrm{Cr}$, excedeu o limite de exposição segura da OMS $\left(2,5\right.$ ng.m $\left.{ }^{-3}\right)$ em FAB em alguns períodos, assim como $\mathrm{Fe}, \mathrm{Sn}, \mathrm{Pb}$ e $\mathrm{As}$.

Os elementos tóxicos são encontrados geralmente em baixas concentrações devido à mobilidade biogeoquímica natural, através das fontes de emissão de poeiras fugitivas não canalizadas de origem natural como a influência eólica, contudo, as atividades antrópicas podem aumentar significativamente a concentração destes elementos pela emissão de poeira canalizada ou não [21], que é o que parece ocorrer na região de estudo.

Com base na presença dos elementos, podese prédizer que a qualidade do ar em IPA encontrase sobre grande influência de fontes estacionárias de 
poluentes atmosféricos (setor industrial), seguindo pelo desgaste de peças de veículos e, finalmente, por fontes naturais. Em FAB, a qualidade do ar parece ser influenciada principalmente pelo tráfego de veículos e emissões industriais na mesma proporção.

\section{Índice da Qualidade do Ar em FAB e IPA}

A qualidade do ar se apresentou como BOA tanto para PTS, quanto para $\mathrm{MP}_{10}$ em ambos os locais durante o período amostrado (FIG. 01 e 02).

No dia 26 de julho, a classificação se apresentou INADEQUADA para o parâmetro $\mathrm{MP}_{10}$ em FAB (IQAR $=111 \%$ ) e em IPA para PTS a classificação foi REGULAR (IQAR=59\%) [13].
Houve outros dias em que a qualidade esteve REGULAR no mês de junho em FAB, para PTS, 09 e 22 de junho IQAR $=69 \%$ e IQAR $=56 \%$, respectivamente. A qualidade do ar se apresentou BOA nos dias 11 e 31 de maio, 27 de julho, 06 de outubro e em todos os dias amostrados de novembro em FAB.

Vale destacar que esta realidade seria diferente se os índices da qualidade do ar para a região fossem baseados nos limites estabelecidos pela OMS, onde o limite máximo de concentração para MP é de $50 \mu \mathrm{g} \cdot \mathrm{m}^{-3}$ [20]. Se assim fosse, para FAB e IPA a qualidade do ar se apresentaria como INADEQUADA na maioria dos dias [13].

Quadro 01: Classificação dos parâmetros da qualidade do ar.

\begin{tabular}{|c|c|c|c|c|c|c|c|c|}
\hline Classificação & Índice & $\begin{array}{c}\text { Níveis de } \\
\text { Cautela sobre } \\
\text { a saúde }\end{array}$ & $\begin{array}{c}P T S \\
24 h \\
\mu g \cdot m^{-3}\end{array}$ & $\begin{array}{c}M P_{10} \\
24 h \\
\mu g \cdot m^{-3}\end{array}$ & $\begin{array}{c}\mathrm{SO}_{2} \\
24 \mathrm{~h} \\
\mu g \cdot \mathrm{m}^{-3}\end{array}$ & $\begin{array}{c}\mathrm{CO} \\
08 \mathrm{~h} \\
\mu \mathrm{g} \cdot \mathrm{m}^{-3}\end{array}$ & $\begin{array}{c}O_{3} \\
01 \mathrm{~h} \\
\mu g \cdot \mathrm{m}^{-3}\end{array}$ & 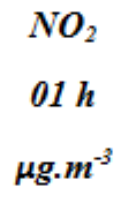 \\
\hline Boa & $0-50$ & & 80 & 50 & 80 & 4,5 & 80 & 100 \\
\hline Regular & $51-100$ & & 240 & 150 & 365 & 9,0 & 160 & 320 \\
\hline Inadequada & $101-199$ & Atenção & 375 & 250 & 800 & 15 & 400 & 1130 \\
\hline Má & $200-299$ & Alerta & 425 & 420 & 1600 & 30 & 800 & 2260 \\
\hline Péssima & $300-399$ & Emergência & 625 & 500 & 2100 & 40 & 1000 & 3000 \\
\hline Crítica & Acima de 400 & Crítica & 875 & 600 & 2620 & 50 & 1200 & 3750 \\
\hline
\end{tabular}

Fonte: US EPA, 2009

Tabela 1: Resumo estatístico das concentrações de PTS $\left(\mu \mathrm{g} \cdot \mathrm{m}^{-3}\right)$ nos pontos em FAB e IPA.

\begin{tabular}{ccccc}
\hline \multirow{2}{*}{ Estação } & \multicolumn{2}{c}{ FAB } & \multicolumn{2}{c}{ IPA } \\
& Média (SD) & Min-Max & Média (SD) & Min-Max \\
\hline Seca & $82,29(37,16)$ & $7-165$ & $70,11(24,51)$ & $44-141$ \\
\hline Transição & $52,40(19,55)$ & $25-81$ & $52,60(12,63)$ & $33-72$ \\
\hline Total & $73,5(35,5)$ & $7-165$ & $63,4(22,5)$ & $33-141$ \\
\hline
\end{tabular}

Tabela 2: Resumo estatístico das concentrações de $\mathrm{MP}_{10}\left(\mu \mathrm{g} \cdot \mathrm{m}^{-3}\right)$ nos pontos em FAB e IPA

\begin{tabular}{ccccc}
\hline \multirow{2}{*}{ Estação } & \multicolumn{2}{c}{ FAB } & \multicolumn{2}{c}{ IPA } \\
& Média (SD) & Min-Max & Média (SD) & Min-Max \\
\hline Seca & $61,00(37,37)$ & $37-167$ & $22,63(8,3)$ & $7-33$ \\
\hline Transição & $25,20(10,96)$ & $12-46$ & $27,10(5,53)$ & $20-38$ \\
\hline Total & $43,95(32,98)$ & $12-167$ & $25,11(7,05)$ & $7-38$ \\
\hline
\end{tabular}


Tabela 3: Coeficiente de Correlação entre as concentrações dos elementos obtidos nas PTS em FAB

\begin{tabular}{|c|c|c|c|c|c|c|c|c|c|c|c|c|c|c|c|c|c|c|c|c|c|c|c|c|c|c|c|c|c|}
\hline & $\mathrm{Al}$ & As & $\mathrm{Br}$ & $\mathrm{Ca}$ & $\mathrm{Cd}$ & $\mathrm{Ce}$ & Co & $\mathrm{Cr}$ & $\mathrm{Cu}$ & $\mathrm{Fe}$ & Hf & $\bar{K}$ & $\mathrm{La}$ & $\mathrm{Mg}$ & $\mathrm{Mn}$ & $\mathrm{Na}$ & $\mathrm{Ni}$ & $\mathrm{Pb}$ & $\mathrm{Sb}$ & $\mathrm{Sc}$ & $\mathrm{Sm}$ & $\mathrm{Sn}$ & $\mathrm{Tb}$ & Th & $\mathrm{Ti}_{\mathrm{T}}$ & $\mathrm{Yb}$ & $\mathrm{Zn}$ & $\mathrm{Zr}$ & PTS \\
\hline $\mathrm{Al}$ & & & & & & & & & & & & & & & & & & & & & & & & & & & & & \\
\hline As & 0,06 & & & & & & & & & & & & & & & & & & & & & & & & & & & & \\
\hline $\mathrm{Br}$ & 0,21 & 0,20 & & & & & & & & & & & & & & & & & & & & & & & & & & & \\
\hline $\mathrm{Ca}$ & 0,89 & 0,05 & 0,04 & & & & & & & & & & & & & & & & & & & & & & & & & & \\
\hline $\mathrm{Cd}$ & $-0,08$ & $-0,25$ & 0,35 & $-0,18$ & & & & & & & & & & & & & & & & & & & & & & & & & \\
\hline $\mathrm{Ce}$ & 0,26 & 0,19 & 0,80 & 0,21 & 0,25 & & & & & & & & & & & & & & & & & & & & & & & & \\
\hline Co & 0,22 & $-0,09$ & 0,71 & 0,21 & 0,31 & 0,88 & & & & & & & & & & & & & & & & & & & & & & & \\
\hline $\mathrm{Cr}$ & 0,23 & 0,37 & 0,84 & 0,11 & 0,17 & 0,86 & 0,73 & & & & & & & & & & & & & & & & & & & & & & \\
\hline $\mathrm{Cu}$ & 0,60 & $-0,06$ & $-0,09$ & 0,70 & 0,06 & $-0,01$ & 0,02 & $-0,07$ & & & & & & & & & & & & & & & & & & & & & \\
\hline $\mathrm{Fe}$ & 0,10 & 0,31 & 0,72 & 0,03 & 0,02 & 0,57 & 0,55 & 0,77 & $-0,08$ & & & & & & & & & & & & & & & & & & & & \\
\hline Hf & 0,15 & 0,54 & 0,37 & 0,14 & 0,16 & 0,66 & 0,45 & 0,49 & $-0,04$ & 0,02 & & & & & & & & & & & & & & & & & & & \\
\hline $\mathrm{K}$ & 0,11 & $-0,09$ & 0,48 & 0,06 & 0,16 & 0,61 & 0,58 & 0,62 & 0,00 & 0,57 & 0,13 & & & & & & & & & & & & & & & & & & \\
\hline $\mathrm{La}$ & 0,12 & $-0,08$ & 0,55 & 0,08 & $-0,06$ & 0,52 & 0,54 & 0,58 & $-0,01$ & 0,71 & $-0,11$ & 0,74 & & & & & & & & & & & & & & & & & \\
\hline $\mathrm{Mg}$ & 0,83 & $-0,04$ & 0,36 & 0,88 & 0,17 & 0,50 & 0,50 & 0,34 & 0,61 & 0,10 & 0,35 & 0,19 & 0,12 & & & & & & & & & & & & & & & & \\
\hline $\mathrm{Mn}$ & 0,67 & $-0,04$ & 0,25 & 0,84 & 0,20 & 0,44 & 0,46 & 0,27 & 0,68 & 0,07 & 0,31 & 0,20 & 0,13 & 0,94 & & & & & & & & & & & & & & & \\
\hline $\mathrm{Na}$ & 0,81 & 0,06 & 0,07 & 0,65 & $-0,17$ & 0,14 & 0,10 & 0,07 & 0,37 & $-0,04$ & 0,12 & 0,00 & 0,05 & 0,57 & 0,35 & & & & & & & & & & & & & & \\
\hline $\mathrm{Ni}$ & 0,36 & 0,09 & 0,42 & 0,35 & 0,53 & 0,40 & 0,39 & 0,43 & 0,49 & 0,27 & 0,27 & 0,29 & 0,23 & 0,50 & 0,58 & $-0,01$ & & & & & & & & & & & & & \\
\hline $\mathrm{Pb}$ & 0,57 & $-0,07$ & $-0,28$ & 0,39 & $-0,19$ & $-0,22$ & $-0,17$ & $-0,24$ & 0,08 & $-0,23$ & $-0,12$ & $-0,17$ & $-0,16$ & 0,20 & 0,02 & 0,65 & $-0,29$ & & & & & & & & & & & & \\
\hline $\mathrm{Sb}$ & 0,13 & 0,22 & 0,64 & 0,16 & 0,30 & 0,84 & 0,82 & 0,64 & $-0,03$ & 0,40 & 0,69 & 0,42 & 0,28 & 0,44 & 0,43 & 0,06 & 0,36 & $-0,19$ & & & & & & & & & & & \\
\hline $\mathrm{Sc}$ & 0,24 & 0,17 & 0,82 & 0,20 & 0,24 & 0,98 & 0,89 & 0,84 & $-0,02$ & 0,61 & 0,60 & 0,64 & 0,58 & 0,48 & 0,43 & 0,13 & 0,37 & $-0,22$ & 0,85 & & & & & & & & & & \\
\hline $\mathrm{Sm}$ & 0,19 & $-0,08$ & 0,67 & 0,10 & 0,01 & 0,58 & 0,50 & 0,66 & 0,00 & 0,70 & $-0,05$ & 0,75 & 0,87 & 0,20 & 0,18 & 0,00 & 0,23 & $-0,17$ & 0,29 & 0,64 & & & & & & & & & \\
\hline Sn & 0,70 & 0,16 & 0,01 & 0,82 & $-0,34$ & 0,14 & 0,07 & 0,12 & 0,71 & 0,18 & $-0,01$ & 0,13 & 0,25 & 0,59 & 0,61 & 0,44 & 0,44 & 0,14 & 0,02 & 0,13 & 0,23 & & & & & & & & \\
\hline $\mathrm{Tb}$ & 0,10 & $-0,05$ & 0,40 & 0,17 & $-0,11$ & 0,37 & 0,45 & 0,34 & 0,05 & 0,61 & $-0,17$ & 0,58 & 0,84 & 0,13 & 0,15 & 0,14 & 0,13 & $-0,11$ & 0,24 & 0,46 & 0,58 & 0,32 & & & & & & & \\
\hline Th & 0,16 & $-0,15$ & 0,81 & 0,06 & 0,49 & 0,89 & 0,87 & 0,74 & $-0,05$ & 0,46 & 0,45 & 0,65 & 0,54 & 0,44 & 0,38 & 0,06 & 0,42 & $-0,25$ & 0,79 & 0,90 & 0,62 & $-0,06$ & 0,36 & & & & & & \\
\hline $\mathrm{Ti}_{\mathrm{i}}$ & 0,04 & 0,35 & $-0,16$ & 0,05 & $-0,27$ & $-0,23$ & $-0,31$ & $-0,21$ & $-0,10$ & $-0,10$ & 0,02 & $-0,40$ & $-0,34$ & $-0,13$ & $-0,16$ & 0,04 & $-0,02$ & 0,11 & $-0,13$ & $-0,22$ & $-0,28$ & 0,16 & $-0,19$ & $-0,37$ & & & & & \\
\hline $\mathrm{Yb}$ & 0,17 & 0,43 & 0,57 & 0,22 & $-0,33$ & 0,48 & 0,36 & 0,54 & 0,01 & 0,79 & 0,07 & 0,38 & 0,69 & 0,14 & 0,13 & 0,11 & 0,07 & $-0,14$ & 0,37 & 0,55 & 0,64 & 0,42 & 0,70 & 0,29 & 0,06 & & & & \\
\hline $\mathrm{Zn}$ & 0,91 & 0,11 & $-0,09$ & 0,82 & $-0,23$ & $-0,02$ & $-0,03$ & $-0,01$ & 0,54 & $-0,03$ & $-0,01$ & $-0,06$ & 0,00 & 0,61 & 0,47 & 0,79 & 0,21 & 0,73 & $-0,08$ & $-0,04$ & 0,00 & 0,72 & 0,04 & $-0,16$ & 0,18 & 0,11 & & & \\
\hline $\mathrm{Zr}$ & 0,18 & $-0,08$ & 0,60 & 0,12 & 0,02 & 0,53 & 0,48 & 0,56 & 0,01 & 0,72 & $-0,16$ & 0,64 & 0,76 & 0,20 & 0,16 & 0,13 & 0,13 & $-0,17$ & 0,25 & 0,56 & 0,75 & 0,23 & 0,70 & 0,53 & $-0,19$ & 0,67 & 0,01 & & \\
\hline PTS & 0,84 & 0,08 & 0,20 & 0,89 & 0,09 & 0,30 & 0,29 & 0,22 & 0,72 & 0,14 & 0,18 & 0,16 & 0,13 & 0,86 & 0,83 & 0,55 & 0,66 & 0,21 & 0,26 & 0,29 & 0,16 & 0,83 & 0,18 & 0,20 & 0,10 & 0,21 & 0,76 & 0,19 & \\
\hline
\end{tabular}

Tabela 4: Coeficiente de Correlação entre as concentrações de elementos obtidos nos PTS em IPA

\begin{tabular}{|c|c|c|c|c|c|c|c|c|c|c|c|c|c|c|c|c|c|c|c|c|c|c|c|c|c|c|c|}
\hline & $\mathrm{Al}$ & As & $\mathrm{Br}$ & $\mathrm{Ca}$ & $\mathrm{Cd}$ & $\mathrm{Ce}$ & Co & $\mathrm{Cr}$ & $\mathrm{Cu}$ & $\mathrm{Fe}$ & Hf & $\bar{K}$ & $\mathrm{La}$ & $\mathrm{Mg}$ & $\mathrm{Mn}$ & $\mathrm{Na}$ & $\mathrm{Ni}$ & $\mathrm{Pb}$ & $\mathrm{Sb}$ & $\mathrm{Sc}$ & $\mathrm{Sm}$ & $\mathrm{Sn}$ & $\mathrm{Sr}$ & Th & $\mathrm{Zn}$ & $\mathrm{Zr}$ & PTS \\
\hline $\mathrm{Al}$ & & & & & & & & & & & & & & & & & & & & & & & & & & & \\
\hline As & 0,40 & & & & & & & & & & & & & & & & & & & & & & & & & & \\
\hline $\mathrm{Br}$ & 0,51 & 0,84 & & & & & & & & & & & & & & & & & & & & & & & & & \\
\hline $\mathrm{Ca}$ & 0,53 & 0,16 & 0,37 & & & & & & & & & & & & & & & & & & & & & & & & \\
\hline $\mathrm{Cd}$ & 0,61 & 0,46 & 0,49 & 0,04 & & & & & & & & & & & & & & & & & & & & & & & \\
\hline $\mathrm{Ce}$ & 0,01 & 0,57 & 0,58 & 0,03 & 0,32 & & & & & & & & & & & & & & & & & & & & & & \\
\hline Co & $-0,03$ & $-0,25$ & 0,00 & 0,43 & $-0,28$ & 0,00 & & & & & & & & & & & & & & & & & & & & & \\
\hline $\mathrm{Cr}$ & 0,59 & 0,71 & 0,62 & 0,06 & 0,77 & 0,39 & $-0,35$ & & & & & & & & & & & & & & & & & & & & \\
\hline $\mathrm{Cu}$ & 0,73 & 0,54 & 0,62 & 0,26 & 0,79 & 0,34 & $-0,27$ & 0,76 & & & & & & & & & & & & & & & & & & & \\
\hline $\mathrm{Fe}$ & 0,50 & 0,78 & 0,77 & 0,34 & 0,51 & 0,54 & $-0,13$ & 0,67 & 0,68 & & & & & & & & & & & & & & & & & & \\
\hline Hf & $-0,14$ & 0,60 & 0,51 & 0,02 & 0,04 & 0,67 & $-0,12$ & 0,26 & 0,06 & 0,45 & & & & & & & & & & & & & & & & & \\
\hline $\mathrm{K}$ & 0,04 & $-0,07$ & 0,15 & 0,50 & $-0,33$ & $-0,05$ & 0,44 & $-0,39$ & $-0,26$ & $-0,08$ & 0,15 & & & & & & & & & & & & & & & & \\
\hline $\mathrm{La}$ & 0,11 & 0,58 & 0,59 & 0,06 & 0,18 & 0,82 & 0,00 & 0,34 & 0,25 & 0,49 & 0,59 & 0,00 & & & & & & & & & & & & & & & \\
\hline $\mathrm{Mg}$ & 0,81 & 0,61 & 0,67 & 0,33 & 0,77 & 0,30 & $-0,21$ & 0,87 & 0,92 & 0,68 & 0,12 & $-0,24$ & 0,22 & & & & & & & & & & & & & & \\
\hline $\mathrm{Mn}$ & 0,78 & 0,66 & 0,75 & 0,43 & 0,71 & 0,36 & $-0,15$ & 0,80 & 0,91 & 0,79 & 0,12 & $-0,19$ & 0,29 & 0,94 & & & & & & & & & & & & & \\
\hline $\mathrm{Na}$ & 0,81 & 0,44 & 0,55 & 0,28 & 0,66 & 0,19 & $-0,13$ & 0,72 & 0,72 & 0,50 & 0,13 & $-0,12$ & 0,13 & 0,84 & 0,74 & & & & & & & & & & & & \\
\hline $\mathrm{Ni}$ & 0,45 & 0,54 & 0,42 & $-0,22$ & 0,81 & 0,41 & $-0,50$ & 0,88 & 0,76 & 0,56 & 0,16 & $-0,60$ & 0,28 & 0,78 & 0,70 & 0,60 & & & & & & & & & & & \\
\hline $\mathrm{Pb}$ & $-0,16$ & 0,04 & $-0,07$ & $-0,06$ & $-0,13$ & 0,03 & $-0,16$ & 0,28 & 0,04 & 0,10 & 0,26 & $-0,19$ & $-0,13$ & 0,18 & 0,07 & 0,15 & 0,23 & & & & & & & & & & \\
\hline $\mathrm{Sb}$ & $-0,14$ & 0,26 & 0,11 & 0,02 & $-0,33$ & 0,06 & $-0,01$ & $-0,13$ & $-0,20$ & 0,02 & 0,24 & 0,05 & 0,17 & $-0,10$ & $-0,05$ & $-0,29$ & $-0,18$ & 0,08 & & & & & & & & & \\
\hline $\mathrm{Sc}$ & 0,79 & 0,64 & 0,76 & 0,45 & 0,65 & 0,27 & $-0,06$ & 0,71 & 0,79 & 0,80 & 0,16 & $-0,10$ & 0,27 & 0,84 & 0,88 & 0,76 & 0,52 & $-0,09$ & $-0,08$ & & & & & & & & \\
\hline $\mathrm{Sm}$ & 0,53 & 0,38 & 0,32 & 0,19 & 0,60 & 0,00 & $-0,22$ & 0,63 & 0,50 & 0,38 & $-0,03$ & $-0,25$ & $-0,10$ & 0,62 & 0,55 & 0,46 & 0,54 & $-0,10$ & $-0,07$ & 0,57 & & & & & & & \\
\hline $\mathrm{Sn}$ & 0,13 & 0,39 & 0,30 & $-0,42$ & 0,41 & 0,47 & $-0,41$ & 0,50 & 0,49 & 0,33 & 0,26 & $-0,53$ & 0,35 & 0,43 & 0,35 & 0,40 & 0,72 & 0,17 & $-0,24$ & 0,19 & 0,11 & & & & & & \\
\hline $\mathrm{Sr}$ & 0,18 & 0,28 & 0,32 & 0,12 & $-0,20$ & 0,05 & $-0,06$ & 0,09 & 0,05 & $-0,06$ & 0,15 & 0,09 & 0,44 & 0,07 & 0,05 & 0,11 & $-0,07$ & $-0,14$ & 0,28 & 0,07 & $-0,01$ & 0,09 & & & & & \\
\hline $\mathrm{Th}$ & 0,70 & 0,49 & 0,69 & 0,63 & 0,49 & 0,19 & 0,17 & 0,55 & 0,58 & 0,71 & 0,15 & 0,17 & 0,20 & 0,65 & 0,75 & 0,66 & 0,27 & $-0,09$ & $-0,20$ & 0,88 & 0,41 & $-0,07$ & $-0,02$ & & & & \\
\hline $\mathrm{Zn}$ & 0,76 & 0,47 & 0,42 & 0,27 & 0,66 & 0,15 & $-0,30$ & 0,81 & 0,78 & 0,52 & 0,09 & $-0,29$ & 0,07 & 0,90 & 0,78 & 0,81 & 0,77 & 0,36 & $-0,09$ & 0,63 & 0,63 & 0,45 & 0,07 & 0,44 & & & \\
\hline $\mathrm{Zr}$ & 0,13 & $-0,15$ & 0,04 & 0,49 & $-0,23$ & $-0,06$ & 0,22 & $-0,30$ & $-0,08$ & $-0,17$ & 0,17 & 0,53 & 0,02 & $-0,07$ & $-0,13$ & 0,13 & $-0,49$ & $-0,13$ & 0,02 & $-0,02$ & $-0,17$ & $-0,31$ & 0,32 & 0,10 & $-0,07$ & & \\
\hline PTS & 0,63 & 0,74 & 0,75 & 0,40 & 0,54 & 0,37 & $-0,10$ & 0,77 & 0,72 & 0,79 & 0,43 & $-0,07$ & 0,26 & 0,84 & 0,83 & 0,79 & 0,58 & 0,33 & 0,03 & 0,81 & 0,41 & 0,34 & 0,07 & 0,74 & 0,75 & 0,02 & \\
\hline
\end{tabular}


Tabela 5: Coeficiente de Correlação entre os níveis de concentrações de elementos obtidos nos $\mathrm{MP}_{10} \mathrm{em}$ FAB.

\begin{tabular}{|c|c|c|c|c|c|c|c|c|c|c|c|c|c|c|c|c|c|c|c|c|c|c|c|c|c|c|}
\hline & $\mathrm{Al}$ & As & $\mathrm{Br}$ & $\mathrm{Ca}$ & $\mathrm{Cd}$ & $\mathrm{Ce}$ & Co & $\mathrm{Cr}$ & $\mathrm{Cu}$ & $\mathrm{Fe}$ & Hf & $\mathrm{K}$ & $\mathrm{La}$ & $\mathrm{Mg}$ & $\mathrm{Mn}$ & $\mathrm{Na}$ & $\mathrm{Ni}$ & $\mathrm{Pb}$ & $\mathrm{Sb}$ & $\mathrm{Sc}$ & $\mathrm{Sm}$ & $\mathrm{Sn}$ & $\mathrm{Ta}$ & Th & $\mathrm{Zn}$ & MP10 \\
\hline $\mathrm{Al}$ & & & & & & & & & & & & & & & & & & & & & & & & & & \\
\hline $\mathrm{As}$ & 0,18 & & & & & & & & & & & & & & & & & & & & & & & & & \\
\hline $\mathrm{Br}$ & 0,39 & 0,70 & & & & & & & & & & & & & & & & & & & & & & & & \\
\hline $\mathrm{Ca}$ & 0,25 & 0,93 & 0,70 & & & & & & & & & & & & & & & & & & & & & & & \\
\hline $\mathrm{Cd}$ & 0,73 & 0,67 & 0,52 & 0,69 & & & & & & & & & & & & & & & & & & & & & & \\
\hline $\mathrm{Ce}$ & 0,29 & $-0,04$ & 0,00 & 0,06 & 0,22 & & & & & & & & & & & & & & & & & & & & & \\
\hline Co & 0,81 & 0,23 & 0,10 & 0,30 & 0,83 & 0,14 & & & & & & & & & & & & & & & & & & & & \\
\hline $\mathrm{Cr}$ & $-0,26$ & 0,82 & 0,53 & 0,75 & 0,17 & $-0,08$ & $-0,29$ & & & & & & & & & & & & & & & & & & & \\
\hline $\mathrm{Cu}$ & $-0,31$ & 0,67 & 0,52 & 0,67 & 0,02 & $-0,22$ & $-0,36$ & 0,88 & & & & & & & & & & & & & & & & & & \\
\hline $\mathrm{Fe}$ & 0,47 & 0,87 & 0,80 & 0,91 & 0,73 & 0,24 & 0,35 & 0,65 & 0,59 & & & & & & & & & & & & & & & & & \\
\hline Hf & 0,31 & $-0,11$ & $-0,02$ & 0,02 & 0,18 & 0,99 & 0,14 & $-0,13$ & $-0,24$ & 0,22 & & & & & & & & & & & & & & & & \\
\hline $\mathrm{K}$ & 0,23 & 0,82 & 0,84 & 0,90 & 0,51 & 0,01 & 0,12 & 0,75 & 0,78 & 0,91 & 0,00 & & & & & & & & & & & & & & & \\
\hline $\mathrm{La}$ & 0,46 & 0,87 & 0,93 & 0,86 & 0,70 & 0,05 & 0,27 & 0,65 & 0,56 & 0,94 & 0,02 & 0,89 & & & & & & & & & & & & & & \\
\hline $\mathrm{Mg}$ & 0,21 & 0,91 & 0,74 & 0,99 & 0,64 & 0,04 & 0,24 & 0,77 & 0,71 & 0,90 & 0,01 & 0,92 & 0,86 & & & & & & & & & & & & & \\
\hline $\mathrm{Mn}$ & 0,36 & 0,83 & 0,86 & 0,86 & 0,56 & $-0,02$ & 0,17 & 0,72 & 0,73 & 0,93 & $-0,03$ & 0,96 & 0,95 & 0,86 & & & & & & & & & & & & \\
\hline $\mathrm{Na}$ & 0,43 & 0,67 & 0,92 & 0,71 & 0,54 & $-0,01$ & 0,16 & 0,50 & 0,50 & 0,77 & $-0,01$ & 0,82 & 0,89 & 0,74 & 0,84 & & & & & & & & & & & \\
\hline $\mathrm{Ni}$ & 0,77 & 0,11 & 0,04 & 0,20 & 0,75 & 0,16 & 0,95 & $-0,40$ & $-0,42$ & 0,28 & 0,18 & 0,05 & 0,20 & 0,13 & 0,11 & 0,13 & & & & & & & & & & \\
\hline $\mathrm{Pb}$ & 0,69 & 0,65 & 0,48 & 0,68 & 0,99 & 0,20 & 0,85 & 0,13 & 0,00 & 0,70 & 0,16 & 0,48 & 0,66 & 0,63 & 0,51 & 0,48 & 0,76 & & & & & & & & & \\
\hline $\mathrm{Sb}$ & 0,41 & 0,72 & 0,85 & 0,68 & 0,50 & $-0,15$ & 0,17 & 0,56 & 0,60 & 0,82 & $-0,15$ & 0,83 & 0,90 & 0,67 & 0,94 & 0,83 & 0,15 & 0,45 & & & & & & & & \\
\hline $\mathrm{Sc}$ & 0,44 & 0,25 & 0,32 & 0,36 & 0,45 & 0,94 & 0,24 & 0,14 & 0,00 & 0,56 & 0,92 & 0,34 & 0,39 & 0,35 & 0,32 & 0,30 & 0,23 & 0,42 & 0,17 & & & & & & & \\
\hline $\mathrm{Sm}$ & 0,68 & 0,18 & 0,09 & 0,20 & 0,57 & 0,51 & 0,62 & $-0,12$ & $-0,23$ & 0,33 & 0,49 & 0,06 & 0,21 & 0,19 & 0,12 & 0,19 & 0,48 & 0,55 & 0,03 & 0,55 & & & & & & \\
\hline $\mathrm{Sn}$ & $-0,11$ & 0,89 & 0,69 & 0,87 & 0,40 & $-0,12$ & $-0,09$ & 0,89 & 0,87 & 0,75 & $-0,17$ & 0,85 & 0,77 & 0,89 & 0,80 & 0,67 & $-0,19$ & 0,38 & 0,64 & 0,16 & $-0,01$ & & & & & \\
\hline $\mathrm{Ta}$ & 0,29 & $-0,11$ & $-0,02$ & 0,01 & 0,16 & 1,00 & 0,11 & $-0,12$ & $-0,24$ & 0,21 & 1,00 & $-0,02$ & 0,02 & 0,00 & $-0,05$ & $-0,02$ & 0,15 & 0,14 & $-0,16$ & 0,92 & 0,49 & $-0,17$ & & & & \\
\hline Th & 0,33 & 0,00 & 0,06 & 0,12 & 0,25 & 1,00 & 0,16 & $-0,05$ & $-0,18$ & 0,31 & 0,99 & 0,08 & 0,12 & 0,10 & 0,05 & 0,06 & 0,18 & 0,23 & $-0,08$ & 0,96 & 0,52 & $-0,07$ & 0,99 & & & \\
\hline $\mathrm{Zn}$ & 0,36 & 0,80 & 0,62 & 0,83 & 0,58 & $-0,24$ & 0,37 & 0,59 & 0,63 & 0,82 & $-0,24$ & 0,83 & 0,76 & 0,81 & 0,85 & 0,61 & 0,28 & 0,58 & 0,76 & 0,07 & 0,14 & 0,67 & $-0,27$ & $-0,17$ & & \\
\hline MP10 & 0,23 & 0,90 & 0,76 & 0,93 & 0,56 & $-0,01$ & 0,16 & 0,84 & 0,79 & 0,92 & $-0,03$ & 0,95 & 0,89 & 0,93 & 0,95 & 0,78 & 0,07 & 0,52 & 0,82 & 0,31 & 0,15 & 0,88 & $-0,04$ & 0,05 & 0,84 & \\
\hline
\end{tabular}

Tabela 6: Coeficiente de Correlação entre os níveis de concentrações de elementos obtidos nos $\mathrm{MP}_{10} \mathrm{em}$ IPA.

\begin{tabular}{|c|c|c|c|c|c|c|c|c|c|c|c|c|c|c|c|c|c|c|c|c|c|c|c|c|c|c|c|}
\hline & As & $\mathrm{Br}$ & $\mathrm{Ca}$ & $\mathrm{Cd}$ & $\mathrm{Ce}$ & $\mathrm{Co}_{0}$ & $\mathrm{Cr}$ & $\mathrm{Cs}$ & $\mathrm{Cu}$ & $\mathrm{Fe}$ & Hf & $\mathrm{K}$ & $\mathrm{La}$ & $\mathrm{Mg}$ & $\mathrm{Mn}$ & $\mathrm{Na}$ & $\mathrm{Ni}$ & $\mathrm{Pb}$ & $\mathrm{Sb}$ & $\mathrm{Sc}$ & $\mathrm{Se}$ & $\mathrm{Sn}$ & $\mathrm{Ta}$ & $\mathrm{Th}$ & $\bar{U}$ & $\mathrm{Zn}$ & MP10 \\
\hline As & & & & & & & & & & & & & & & & & & & & & & & & & & & \\
\hline $\mathrm{Br}$ & 0,60 & & & & & & & & & & & & & & & & & & & & & & & & & & \\
\hline $\mathrm{Ca}$ & 0,52 & 0,40 & & & & & & & & & & & & & & & & & & & & & & & & & \\
\hline $\mathrm{Cd}$ & 0,03 & $-0,05$ & $-0,03$ & & & & & & & & & & & & & & & & & & & & & & & & \\
\hline $\mathrm{Ce}$ & 0,69 & 0,54 & 0,15 & 0,13 & & & & & & & & & & & & & & & & & & & & & & & \\
\hline Co & $-0,04$ & 0,41 & 0,05 & 0,04 & 0,01 & & & & & & & & & & & & & & & & & & & & & & \\
\hline $\mathrm{Cr}$ & 0,46 & 0,43 & $-0,05$ & 0,10 & 0,71 & 0,24 & & & & & & & & & & & & & & & & & & & & & \\
\hline $\mathrm{Cs}$ & 0,47 & 0,64 & 0,41 & 0,28 & 0,25 & 0,67 & 0,12 & & & & & & & & & & & & & & & & & & & & \\
\hline $\mathrm{Cu}$ & $-0,04$ & $-0,14$ & $-0,29$ & 0,85 & 0,12 & $-0,06$ & 0,03 & 0,12 & & & & & & & & & & & & & & & & & & & \\
\hline $\mathrm{Fe}$ & 0,85 & 0,62 & 0,32 & 0,28 & 0,83 & $-0,08$ & 0,66 & 0,36 & 0,14 & & & & & & & & & & & & & & & & & & \\
\hline $\mathrm{Hf}$ & 0,16 & 0,25 & 0,33 & $-0,02$ & $-0,29$ & 0,43 & $-0,23$ & 0,63 & $-0,18$ & $-0,09$ & & & & & & & & & & & & & & & & & \\
\hline $\mathrm{K}$ & 0,63 & 0,49 & 0,80 & $-0,21$ & 0,21 & 0,31 & 0,30 & 0,49 & $-0,42$ & 0,36 & 0,44 & & & & & & & & & & & & & & & & \\
\hline $\mathrm{La}$ & 0,00 & 0,47 & 0,14 & 0,13 & 0,08 & 0,91 & 0,14 & 0,76 & $-0,05$ & 0,02 & 0,42 & 0,25 & & & & & & & & & & & & & & & \\
\hline $\mathrm{Mg}$ & 0,51 & 0,59 & 0,87 & 0,09 & 0,35 & 0,12 & 0,20 & 0,37 & $-0,14$ & 0,43 & 0,13 & 0,71 & 0,20 & & & & & & & & & & & & & & \\
\hline $\mathrm{Mn}$ & 0,67 & 0,49 & 0,72 & 0,38 & 0,52 & 0,32 & 0,48 & 0,62 & 0,13 & 0,64 & 0,21 & 0,73 & 0,36 & 0,76 & & & & & & & & & & & & & \\
\hline $\mathrm{Na}$ & 0,39 & 0,58 & 0,28 & 0,00 & 0,42 & $-0,28$ & 0,02 & 0,09 & $-0,05$ & 0,46 & $-0,05$ & 0,05 & $-0,10$ & 0,46 & 0,06 & & & & & & & & & & & & \\
\hline $\mathrm{Ni}$ & 0,22 & 0,47 & 0,15 & 0,54 & 0,37 & 0,01 & 0,14 & 0,19 & 0,51 & 0,43 & $-0,19$ & $-0,12$ & 0,09 & 0,46 & 0,30 & 0,54 & & & & & & & & & & & \\
\hline $\mathrm{Pb}$ & 0,05 & 0,13 & $-0,05$ & 0,90 & 0,35 & 0,05 & 0,25 & 0,17 & 0,79 & 0,37 & $-0,26$ & $-0,27$ & 0,14 & 0,23 & 0,37 & 0,22 & 0,76 & & & & & & & & & & \\
\hline $\mathrm{Sb}$ & $-0,29$ & 0,21 & 0,02 & 0,05 & $-0,29$ & 0,92 & $-0,11$ & 0,62 & $-0,06$ & $-0,35$ & 0,54 & 0,15 & 0,89 & 0,00 & 0,13 & $-0,35$ & $-0,08$ & $-0,02$ & & & & & & & & & \\
\hline $\mathrm{Sc}$ & $-0,31$ & 0,19 & 0,02 & 0,05 & $-0,30$ & 0,91 & $-0,14$ & 0,61 & $-0,06$ & $-0,37$ & 0,53 & 0,14 & 0,89 & $-0,01$ & 0,11 & $-0,34$ & $-0,09$ & $-0,03$ & 1,00 & & & & & & & & \\
\hline $\mathrm{Se}$ & 0,50 & 0,49 & 0,17 & $-0,06$ & 0,77 & 0,05 & 0,64 & 0,21 & $-0,17$ & 0,70 & $-0,28$ & 0,22 & 0,18 & 0,27 & 0,40 & 0,36 & 0,04 & 0,11 & $-0,18$ & $-0,19$ & & & & & & & \\
\hline $\mathrm{Sn}$ & 0,03 & $-0,02$ & $-0,20$ & 0,94 & 0,25 & $-0,05$ & 0,22 & 0,12 & 0,92 & 0,32 & $-0,26$ & $-0,35$ & 0,00 & 0,02 & 0,28 & 0,06 & 0,64 & 0,94 & $-0,10$ & $-0,11$ & 0,01 & & & & & & \\
\hline $\mathrm{Ta}$ & 0,06 & 0,16 & 0,37 & $-0,15$ & $-0,41$ & 0,33 & $-0,34$ & 0,52 & $-0,32$ & $-0,18$ & 0,96 & 0,41 & 0,35 & 0,09 & 0,12 & $-0,08$ & $-0,30$ & $-0,41$ & 0,50 & 0,49 & $-0,27$ & $-0,40$ & & & & & \\
\hline Th & 0,53 & 0,19 & 0,44 & $-0,14$ & 0,00 & $-0,24$ & $-0,12$ & 0,27 & $-0,23$ & 0,27 & 0,69 & 0,47 & $-0,21$ & 0,20 & 0,22 & 0,22 & $-0,15$ & $-0,34$ & $-0,20$ & $-0,22$ & $-0,10$ & $-0,29$ & 0,70 & & & & \\
\hline $\mathrm{U}$ & $-0,01$ & $-0,04$ & $-0,16$ & 0,72 & 0,39 & $-0,12$ & 0,08 & 0,04 & 0,65 & 0,26 & $-0,34$ & $-0,40$ & $-0,01$ & 0,03 & 0,14 & 0,18 & 0,56 & 0,82 & $-0,13$ & $-0,12$ & 0,12 & 0,73 & $-0,46$ & $-0,31$ & & & \\
\hline $\mathrm{Zn}$ & 0,89 & 0,52 & 0,43 & 0,02 & 0,72 & 0,02 & 0,64 & 0,39 & $-0,10$ & 0,82 & 0,20 & 0,63 & 0,03 & 0,42 & 0,66 & 0,31 & 0,04 & 0,02 & $-0,25$ & $-0,27$ & 0,60 & 0,00 & 0,10 & 0,52 & $-0,06$ & & \\
\hline MP10 & 0,62 & 0,52 & 0,85 & 0,10 & 0,37 & 0,30 & 0,34 & 0,55 & $-0,15$ & 0,47 & 0,38 & 0,90 & 0,30 & 0,83 & 0,87 & 0,18 & 0,11 & 0,08 & 0,15 & 0,13 & 0,32 & $-0,05$ & 0,33 & 0,38 & $-0,08$ & 0,65 & \\
\hline
\end{tabular}


Figura 01: Distribuição percentual das classes de acordo com o IQAr quanto a PTS e $\mathrm{MP}_{10}$ em FAB.

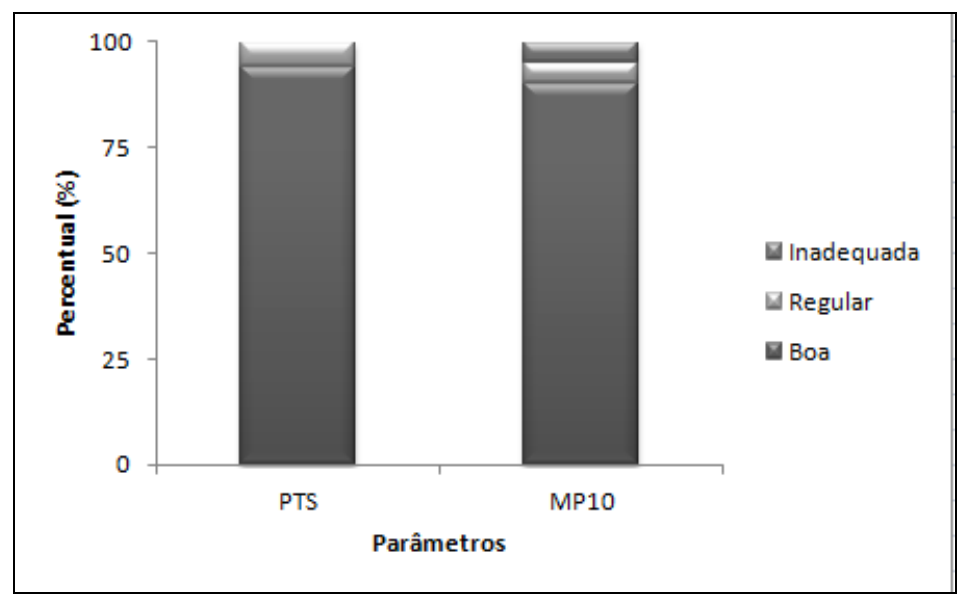

Figura 02: Distribuição percentual das classes de acordo com o IQAr quanto PTS e MP ${ }_{10}$ em IPA.

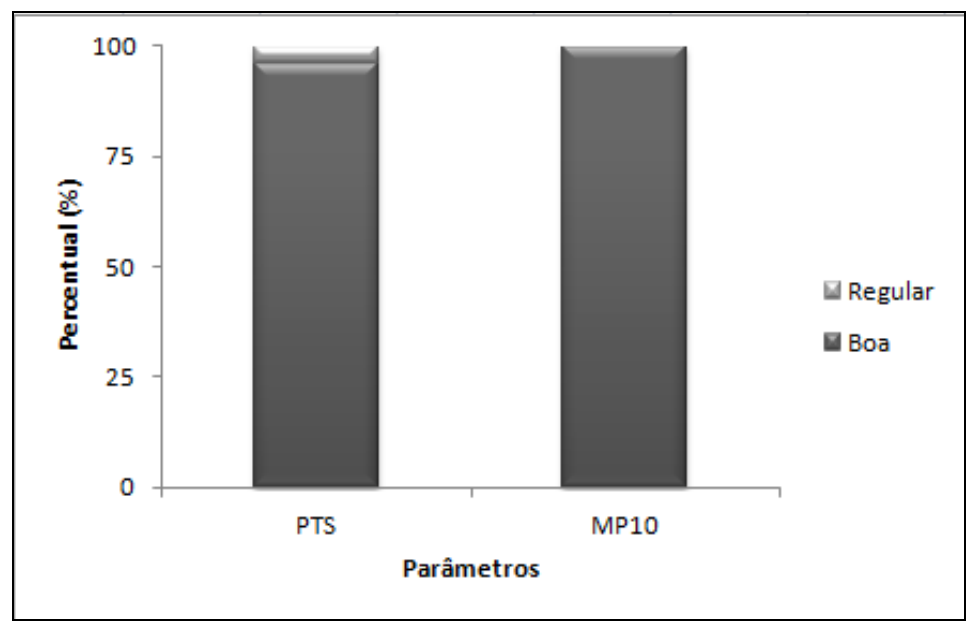




\section{CONCLUSÕES}

Em ambos os pontos, foram encontradas concentrações significativas de MP, mas em sua maioria se apresentaram em concordância com a Resolução CONAMA n ${ }^{\circ}$ 03/90, enquanto que em relação à OMS, a qualidade do ar seria classificada como REGULAR ou INADEQUADA por várias vezes. A atmosfera na RMVA mostrou quantidade significativa de elementos tóxicos e oligo-elementos que podem ser advindos de indústrias, principalmente do seguimento siderúrgico, bem como tráfego de veículos.

\section{AGRADECIMENTOS}

Agradecemos à CAPES, ao Sr. Leandro Coelho Dalvi do Departamento de Meio Ambiente e Qualidade de CENIBRA S.A, ao Laboratório de Análise por Ativação Neutrônica, CDTN / CNEN BH/MG e ao UNILESTE.

\section{REFERÊNCIAS}

[1] BRASIL. Lei Complementar $n^{\circ} 51$, de 30 de dezembro de 1998 do Estado de Minas Gerais. Institui a Região Metropolitana do Vale do Aço, dispõe sobre sua organização e funções e dá outras providências.

[2] IBGE - Instituto Brasileiro de Geografia e Estatística. População Total Residente - Unidade de Federação e Municípios - 2010. Disponível em: < http://www.censo2010.ibge.gov.br/sinopse/index.ph $\mathrm{p}$ ? uf=31\&dados $=1>$. Acesso em: 12 de junho de 2011.

[3] ENERGÉTICA Qualidade do Ar, DIAS, J.W.C. Manual de Operação do Amostrador de Grandes Volumes para Partículas de até $10 \mu \mathrm{m}$ (AGV $\left.\mathrm{MP}_{10}\right)$. Rio de Janeiro, 2005.

[4] ENERGÉTICA Qualidade do Ar, DIAS, J.W.C. Manual de Operação do Amostrador de Grandes Volumes para Partículas Totais em Suspensão (AGV - PTS). Rio de Janeiro, 2009.

[5] US EPA - UNITED STATES ENVIRONMENTAL PROTECTION AGENCY. Air Quality Criteria for Particulate Matter. Vol. 2.
EPA/600/P-99/002a-f, 2004. Disponível em: <www.epa.gov/pmresearch/> Acesso em: 28 mar. 2010.

[6] EWING, G.W. Métodos Instrumentais de Análise Química. vol. II, Ed. Edgard Blucher LTDA, São Paulo, 1972.

[7] BOUÇAS, J. G. Aplicação de técnicas nucleares nos estudos e avaliação da poluição do ar na Região Metropolitana de Belo Horizonte. 2009. Dissertação (Mestrado) Comissão Nacional de Energia Nuclear, Belo Horizonte, 2009.

[8] AVINO, P., GAPANNESI, G., ROSADA, A. Heavy metal determination in atmospheric particulate matter by Instrumental Neutron Activation Analysis. Microchemical Journal, 2008, $97-106$.

[9] QUEIROZ, M.T.A. Bioacumulação de Metais Pesados do Rio Piracicaba, Minas Gerais, Aplicando a Análise por Ativação Neutrônica Instrumental. Dissertação (Mestrado). Centro Universitário do Leste de Minas Gerais - UnilesteMG: 2006.

[10] AMATO, F. et al. Spatial and chemical patterns of $\mathrm{PM}_{10}$ in road dust deposited in urban environment. Atmospheric Environment, Oxford, Vol. 43, p. 1650-1659, 2009.

[11] POOLEY, D.F, MILLE M. Air pollution and health: composition of air pollution particles. Cardiff: University of Wales, 1999. Cap.28.

\section{[12] CANCIO J.L., CASTELLANO A.V.,} HERNÁNDEZ M.C., BETHENCOURT R.G., ORTEGA E.M. Metallic Species in Atmospheric Particulate Matter in Las Palmas de Gran Canaria. Journal of Hazardous Materials 160 (2008) 521-528. Science Direct.

[13] PULINO, A. M.; SANTI, A. M. M. Siderurgia e Poluição Atmosférica: Um Estudo de Caso - A Qualidade do Ar No Município de Ipatinga, Região do Vale do Aço, Minas Gerais. In: CONGRESSO BRASILEIRO DE ENGENHARIA SANITÁRIA E AMBIENTAL, 24, 2007, Belo Horizonte. Anais eletrônicos. Disponível em: <http://www.abes.locaweb.com.br/XP/XP-

EasyPortal/Site/XP PortalPaginaShow.php?id=376> . Acesso em: 14 mar. 2010. 
[14] SILVA C.N.C. Poluição do Ar. Ambiente Brasil. Disponível em:

<http://www.ambientebrasil.com.br/composer.php3? base $=$./urbano/index.html\&conteudo=./urbano/artigo s/qualidade_ar.html>. Acesso em: 03 mar. 2010.

[15] GOYAL, S. Effect of Winds on $\mathrm{SO}_{2}$ and Suspended Particulate Matter Concentrations in Delhi. Atmospheric Enviroment 36 (2002) 29252930.

[16] BILOS C., Colombo J. C., SKORUPKA C. N., Rodriguez Presa M. J. Sources, Distribution and Variability of Airborne Trace Metals in La Plata City Area, Argentina. Environmental Pollution, 2001, 149-158.

[17] MORENO-GRAU, S.; PEHREZ-TORNELL, A.; BAYO, J.; MORENO, J.; ANGOSTO, J.M.; MORENO, J. Particulate Matter and Heavy Metals in the Atmospheric Aerosol from Cartagena, Spain. Atmospheric Environment 34 (2000) 5161-5167.

[18] QUEROL, X. et al. Source origin of trace elements in PM from regional background, urban and industrial sites of Spain. Atmospheric Environment, New York, Vol. 41, p. 7219-7231, 2007.

[19] MAGALHÃES, L.C. Estudo do Material Particulado e Metais associados às Partículas Totais em suspensão na Cidade de Ouro Preto, MG. Dissertação (mestrado), Universidade Federal de Outro Preto, Instituto de Ciências Exatas e Biológicas. Ouro Preto: 2005.

[20] WHO - World Health Organization. Air Quality Guidelines for Europe. Copenhagen: WHO Regional Publication n`91, 2006. 28p.

[21] DAVIS, W.T. Air Pollution Engineering Manual. Air and Waste Management Association. Second Edition. Canada, 2000. 